\title{
Specific heat of Bosons in a lattice
}

\author{
R. Ramakumar ${ }^{a, b}$ and A. N. Das ${ }^{a}$ \\ ${ }^{a}$ Condensed Matter Physics Group, Saha Institute of Nuclear Physics, 1/AF Bidhannagar, Kolkata-700064, INDIA \\ ${ }^{b}$ Department of Physics and Astrophysics, University of Delhi, Delhi-11000\%, INDIA
}

(17 August 2005)

\begin{abstract}
We present a theoretical study of specific heat of bosons $\left(C_{v}\right)$ in a simple cubic lattice. We have studied the non-interacting bosons and the Tonks gas. For both cases, the $C_{v}$ above the bose condensation temperature shows considerable temperature dependence compared to that of free bosons. For Tonks gas, we find that the low-temperature specific heat increases as the system gets closer to the Mott transition.
\end{abstract}

PACS numbers: 03.75.Lm, 03.75.Nt, 03.75.Hh, 67.40.-w

\section{INTRODUCTION}

Investigations of collective properties of bosons in optical lattices produced by counter propagating laser beams is now a rapidly expanding field of research. It was shown ${ }^{1}$ early on that bosons in optical lattices can be adequately modeled by employing a Bose-Hubbard model. Properties of a system modeled by this model depends on lattice symmetry, inter-site boson hopping energy $(t)$, on-site boson-boson interaction strength $(U)$, and the number of bosons per site $(n)$. Experimentalists have achieved great control over several of these parameters by changing the characteristic of the laser beams and their relative orientations. Recently, Greiner and collaborators $^{2}$ demonstrated bose-condensed to Mott insulator transition in a system of interacting bosons in a simple cubic optical lattice. This transition was predicted in theoretical studies ${ }^{1,3-7}$ on Bose-Hubbard model.

For a system of repulsive interacting bosons living in a lattice, bosons will try to avoid multiple occupancy of the sites. When the number of bosons per site is less than or equal to unity and in the strong interaction limit $(U>>t)$ double (or multiple) occupancy of any site is forbidden. In this case, one has a quantum version of Tonks gas $^{8}$ (here after referred to as Tonks gas) in a lattice. One dimensional Tonks gas was investigated by Girardeau ${ }^{9}$ long time back. Quite recently TonksGirardeau gas $^{9,10}$ regime has been achieved in experimental studies ${ }^{11}$ of interacting bosons in one-dimensional optical lattices. It is reasonable to expect that Tonks gas in two and three dimensional lattice will be achieved in the near future. Thermodynamic properties of bosons in a lattice is of considerable interest in this context. In this short communication we present a theoretical study of specific heat of non-interacting and strongly interacting bosons in a simple cubic (sc) lattice. As should be clear by now, the experiments mentioned above and experiments that would be conducted in future are our primary motivation for the work presented in this paper. Heat capacity measurements on an optically trapped, strongly interactiong Fermi gas of atoms has been reported recently ${ }^{12}$ and one can expect that such measurements for bosons in an optical lattice will be done in the near future. The effects of the presence of the harmonic confining potential is not included in our calculations given below. Our results for the non-interacting bosons would be reasonable for a trap of shallow confining potential. It may be mentioned, however, that the strongly interacting regime (Tonks limit) could be reached in experiments with extremely deep optical lattice potential. This, in turn, creates a deep harmonic trap, generated by the envelope of the laser beams. For realistic calculations of experimentally measured quantities in the Tonks limit at present, one should take account of the harmonic trap potential. Our study in the Tonks limit may be considered as a starting point of a more complete calculation where the effects of inhomogeneties produced by the confining potenetial is included.

\section{SPECIFIC HEAT OF LATTICE BOSONS}

The specific heat can be obtained from the energy given by

$$
E=\int_{-W}^{W} \epsilon \rho(\epsilon) n_{B}(\epsilon) d \epsilon
$$

where $\rho(\epsilon)$ is the normalized single boson Density of States (DOS) of a band of width $2 W, n_{B}(\epsilon)=$ $1 /\left[\exp (\beta(\epsilon-\mu)-1]\right.$ with $\beta=1 / k_{B} T$ where $k_{B}$ is the Boltzmann's constant and $T$ is the temperature, and $\mu$ is the chemical potential.

First we consider the case of $T>T_{B}$, where $T_{B}$ is the Bose condensation temperature. In this case, the chemical potential is below the band bottom and is temperature dependent. The chemical potential and the bose condensation temperature are obtained by solving the number equation

$$
n=\int_{-W}^{W} \rho(\epsilon) n_{B}(\epsilon) d \epsilon
$$

where $n$ is the number of bosons per site. For $T>T_{B}$, the energy is given by Eq. (1) and we obtain the constant volume specific heat (per site) to be 


$$
C_{v}=\frac{1}{k_{B} T^{2}}\left(I_{3}-\frac{I_{2} I_{1}}{I_{0}}\right)
$$

where,

$$
\begin{aligned}
& I_{0}=\int_{-W}^{W} F(\epsilon-\mu) \rho(\epsilon) d \epsilon, \\
& I_{1}=\int_{-W}^{W} \epsilon F(\epsilon-\mu) \rho(\epsilon) d \epsilon, \\
& I_{2}=\int_{-W}^{W}(\epsilon-\mu) F(\epsilon-\mu) \rho(\epsilon) d \epsilon, \\
& I_{3}=\int_{-W}^{W} \epsilon(\epsilon-\mu) F(\epsilon-\mu) \rho(\epsilon) d \epsilon .
\end{aligned}
$$

In the above equations,

$$
F(\epsilon-\mu)=\frac{e^{\beta(\epsilon-\mu)}}{\left[e^{\beta(\epsilon-\mu)}-1\right]^{2}} .
$$

Now we consider the case of $T<T_{B}$. In this case the chemical potential is temperature independent and is pinned to the bottom of the band $(-W)$. The band bottom has a macroscopic number of particles (the Bose condensate) and the rest of the particles are in the excited states. Denoting $n_{\text {ext }}$ as the number of bosons (per site) in excited states, the energy per site is

$$
E=\int_{-W}^{W} \epsilon \rho(\epsilon) n_{B}(\epsilon) d \epsilon-W\left(n-n_{e x t}\right) .
$$

The specific heat in this case becomes,

$$
C_{v}=\frac{1}{k_{B} T^{2}} \int_{-W}^{W}(\epsilon+W)^{2} \rho(\epsilon) F(\epsilon+W) d \epsilon .
$$

Non-interacting bosons: The Hamiltonian of the noninteracting bosons in a tight-binding energy band is:

$$
H=\sum_{\mathbf{k}}[\epsilon(\mathbf{k})-\mu] c_{\mathbf{k}}^{\dagger} c_{\mathbf{k}},
$$

where, $\epsilon_{s c}\left(k_{x}, k_{y}, k_{z}\right)=-2 t \sum_{\nu=x}^{z} \cos \left(k_{\nu}\right)$ is the energy band structure of a single boson in a sc lattice with lattice constant set to unity, $c_{\mathbf{k}}=\sqrt{1 / N_{S}} \sum_{i} c_{i} \exp \left(i \mathbf{k} \cdot \mathbf{R}_{\mathbf{i}}\right)$, $c_{i}$ is the annihilation operator of a boson at site $i$, and $N_{S}$ is the number of lattice sites.

Strongly interacting bosons (the Tonks gas limit): To study the Tonks gas limit, we start from the BoseHubbard model given by

$$
H=-t \sum_{<i j>}\left(c_{i}^{\dagger} c_{j}+h . c .\right)+\frac{U}{2} \sum_{i} n_{i}\left(n_{i}-1\right)-\mu \sum_{i} n_{i},
$$

where $n_{i}=c_{i}^{\dagger} c_{i}$. In the above Hamiltonian $c_{\mathbf{i}}^{\dagger}\left(c_{i}\right)$ is the boson creation (annihilation) operator, $U$ the bosonboson repulsive interaction energy. The Tonks gas regime is achieved in the strong correlation limit $(U / t>>1)$ when the number of particles per site $n=N / N_{s}$ is restricted to be less than or equal to unity. In this limit, the double (or multiple) occupancies of the sites are forbidden. We will follow a Renormalized Hamiltonian Approach (RHA) similar to that used for strongly interacting fermions ${ }^{13,14}$ based on Gutzwiller approximation ${ }^{15}$. In this method, the strict constraint of no double occupancy shows up in the renormalization factor for hopping. The hopping (or band) renormalization factor is obtained by taking the ratio of hopping probability between two sites in the correlated space to that in the non-correlated space. The hopping probability in the projected space (of no multiple occupancy) is

$$
p_{\text {corr }}=n(1-n) \text {. }
$$

The above equation means that the site from which hopping takes place should be occupied and the target site should be empty in the projected space. To find the hopping probability in the non-correlated state, we have to first find the probability that a site is occupied. The occupancy of the site to which a boson hops is irrelevant since we are dealing with bosons. To find the site occupancy probability of a site from which a boson hops, we employ the following route. First we find the number of ways $N_{B}$ number of bosons can be distributed in $N_{S}$ number of lattice sites and then the number of ways of distributing the same number of bosons in $\left(N_{S}-1\right)$ number of lattice sites. The difference would give the number of configurations where a particular site among $N_{S}$ number of lattice sites is occupied by bosons. The number of configurations possible for $N_{B}$ bosons on $N_{S}$ sites is given by

$$
w\left(N_{B}: N_{S}\right)=\frac{\left(N_{B}+N_{S}-1\right) !}{N_{B} !\left(N_{S}-1\right) !} .
$$

The number of configurations possible for $N_{B}$ bosons on $\left(N_{S}-1\right)$ sites is given by

$$
w\left(N_{B}: N_{S}-1\right)=\frac{\left(N_{B}+N_{S}-2\right) !}{N_{B} !\left(N_{S}-2\right) !} .
$$

The probability that a particular site is occupied is then

$$
p_{\text {occ }}=1-\frac{w\left(N_{B}: N_{S}-1\right)}{w\left(N_{B}: N_{S}\right)} .
$$

In the thermodynamic limit,

$$
p_{o c c}=\frac{n}{1+n} .
$$

As mentioned for non-interacting bosons, the hopping takes place irrespective of the target site being empty or occupied by any number of bosons. Hence the hopping probability for the non-interacting case is 


$$
p_{\text {nocorr }}=\frac{n}{1+n}
$$

The band-width renormalization factor $\left(p_{\text {corr }} / p_{\text {nocorr }}\right)$ then is,

$$
\phi_{B}=1-n^{2}
$$

Using the above $\phi_{B}$ the renormalized Hamiltonian valid in the strong coupling limit and for $n \leq 1$ is,

$$
H_{s c}=\sum_{\mathbf{k}}\left[\phi_{B}(n) \epsilon(\mathbf{k})-\mu\right] c_{\mathbf{k}}^{\dagger} c_{\mathbf{k}} .
$$

The above is the Hamiltonian of Tonks gas on a lattice. One can immediately see that the band width is strongly $n$ dependent. For $n=1$, the effective mass diverges and the system is a Bose-Mott-Hubbard insulator. In passing, we note that for the fermion Hubbard model, the corresponding hopping renormalization factor $^{13}$ is $2(1-n) /(2-n)$. We do realize that the RHA approach based on Gutzwiller approximation used to obtain Eq. 20 has its limitations. Within this approach, the effect of strong interaction shows up only in the enhanced effective mass of the bosons. This approximation would be reasonable for large connectivity (i.e., for large number of nearest neighbors). One can get some feel for the accuracy of the approximation by comparing with the same for fermions. In the case fermions, it has been shown that the Gutzwiller approximation gets progressively better as the dimensionality increases and becomes exact in infinite dimensions and that in three dimensions it is reasonable ${ }^{16}$. For strongly interacting bosons also, the Gutzwiller wave function is believed to be exact for large dimensionality ${ }^{17}$ and can be expected to be reasonable in three dimensions.

As mentioned earlier the chemical potential and the Bose condensation temperature $\left(T_{B}\right)$ is determined by solving the number equation. Results of this calculations, for bosons in a sc lattice, are shown in Fig. 1. For the non-interacting bosons, the $T_{B}$ shows an initial fast rise and then almost a linear increase with $n$. In the case of strongly interacting bosons, correlation-effects lead to an enhancement of the boson effective mass with increasing band-filling. This increase in the effective mass becomes dominant beyond $n=0.5$. As a consequence the $T_{B}$ starts decreasing and finally going to zero at integer filling for which the transition to Bose-Mott-Hubbard insulating state takes place. It may be noted that the $T_{B}$ for strongly interacting bosons is almost the same as that for non-interacting bosons for low filling $(n<0.2)$. This is a signature of the fact that correlation effects are not significant for low density. The specific heat is calculated using the Eqs. (3) and (10). For the Tonks gas calculations, the original band-width is replaced by the renormalized band-width. The results for non-interacting bosons in a sc lattice and a comparison with that for strongly interacting bosons are shown in Fig. 2. In general, the specific heat increases with temperature for $T<T_{B}$, shows a $\lambda$ anomaly at $T_{B}$ and then decreases with temperature. For non-interacting bosons, the specific heat (per site) follows the same curve at low temperatures irrespective of the band filling. The peak value of the specific heat, however, depends on the filling. The peak value is larger for higher filling. It is also found that the peak value does not depend on the bandwidth. For strongly interacting bosons Fig.2 shows that for a fixed boson density (i) the peak in the specific heat occurs at a temperature lower than that for the non-interacting bosons, (ii) the peak value, however, remains the same for both interacting and non-interacting cases. The latter result is a consequence of the fact that the peak value of the specific heat depends only on the boson density and not on the boson effective mass (or bandwidth). The first behavior is a combined effect of the reduced value of $T_{B}$ for interacting bosons compared to the non-interacting bosons at the same density and the appearance of the specific heat peak at $T_{B}$. As mentioned previously the effect of the on-site interation is small for low boson density and becomes increasingly dominant as the boson density approaches to integer filling, the difference between the specific heat curves as well as between the peak temperatures for the interating and non-interacting bosons is small for $n=0.4$ and is large for $n=0.8$ where the correlation effect is dominant. In experiments it is now possible to tune the hopping of bosons for a given density of bosons to control the effective interaction strength and to obtain results for weakly (or non-interacting) bosons and strongly interacting bosons. The ratio of the peak temperatures of the specific heat for the non-interacting and interacting cases for a fixed boson density may serve as a measure of effective interaction. A comparison of specific heats for non-interacting bosons in a sc lattice and that for bosons in a 3-d box is shown in Fig. 3. The specific heat for free bosons in a 3-d box is obtained by using the DOS $\rho(\epsilon) \propto \sqrt{\epsilon}$. For the sc case the specific heat follows a S-shaped curve for $T<T_{B}$ and it shows a considerable temperature dependence above $T_{B}$ compared to that of free bosons. The $\lambda$ anomaly is very prominent for the sc case in comparison with the free bosons. The results for strongly interacting bosons are shown in Fig. 4. For the strongly interacting bosons, it is seen that with increasing $n$ the specific heat versus temperature curves become narrower. At a low temperature the specific heat as well as the slope of the specific heat with temperature increases with increasing $n$. This is because of increasing effective mass of the bosons with $n$.

\section{CONCLUSIONS}

In this paper we have presented specific heat for bosons in a tight-binding band corresponding to a sc lattice. We investigated non-interacting bosons and Tonks gas. In both cases, the specific heat is found to have consider- 
ably more temperature dependence above $T_{B}$ compared to that of free bosons in a box. For the strongly interacting bosons, we find that the low-temperature specific heat gets enhanced as the systems moves towards the Mott transition.

${ }^{b}$ on lien from SINP, Kolkata.

${ }^{1}$ D. Jaksch, C. Bruder, J. I. Cirac, C. W. Gardiner, and P. Zoller, Phys. Rev. Lett. 81, 3108 (1998).

${ }^{2}$ M. Greiner, O. Mandel, T. Esslinger, T. W. Hänsch, and I. Bloch, Nature 415, 39 (2002).

${ }^{3}$ M. P. A. Fisher, P. B. Weichman, G. Grinstein, and D. S. Fisher, Phys. Rev. B 40, 546 (1989).

${ }^{4}$ W. Krauth and N. Trivedi, Europhys. Lett. 14, 627 (1991).

${ }^{5}$ K. Sheshadri, H. R. Krishnamurthy, R. Pandit, and T. V. Ramakrishnan, Europhys. Lett. 22, 257 (1993).

${ }^{6}$ J. K. Freericks and H. Monien, Phys. Rev. B 53, 2691(1996).

${ }^{7}$ D. Van Oosten, P. van der Straten, and H. T. C. Stoof, Phys. Rev. A 63, 054601(2001).

${ }^{8}$ L. Tonks, Phys. Rev. 50, 955 (1936).

${ }^{9}$ M. Girardeau, J. Math. Phys. 1, 1605 (1960).

${ }^{10}$ E. H. Lieb and W. Liniger, Phys. Rev. 130, 1605 (1963).

11 B. Paredes, A. Widera, V. Murg, O. Mandel, S. Fölling, I. Cirac, G. V. Shlyapnikov, T. W. Hänsch, and I. Bloch, Nature 429, 277 (2004).

12 J. Kinast, A. Turlapov, J. E. Thomas, Qijin Chen, Jelena Stajic and K. Levin, Science 307, 1296, 2005.

${ }^{13}$ F. C. Zhang, C. Gros, T. M. Rice, and H. Shiba, Supercond. Sci. Technol. 1, 36(1988).

14 A. N. Das, J. Konior, A. M. Oles, and D. K. Ray, Phys. Rev. B 44, 7680 (1991).

${ }^{15}$ M. C. Gutzwiller, Phys. Rev. Lett. 10, 159 (1963).

${ }^{16}$ A. Georges, G. Kotliar, W. Krauth, and M. J. Rozenberg, Rev. Mod. Phys. 68, 13 (1996).

${ }^{17}$ D. S. Rokhsar and B. G. Kotliar, Phys. Rev. B 44, 10328 (1991).

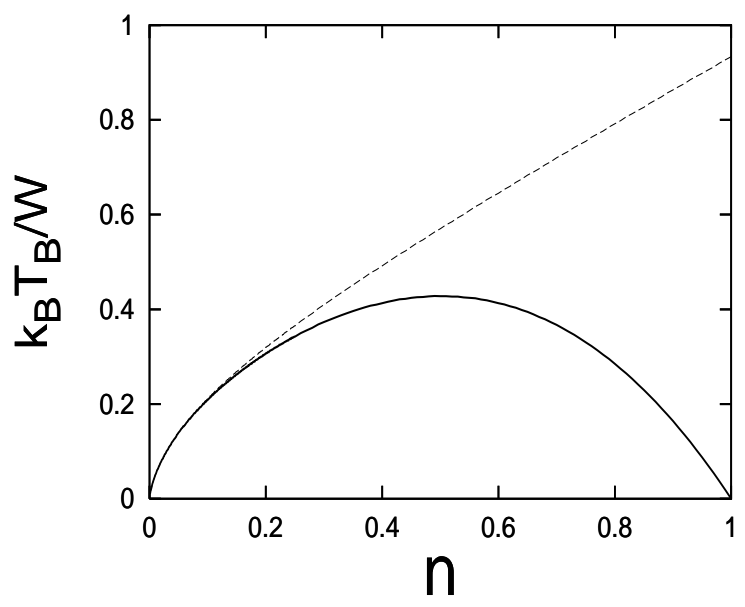

FIG. 1. Bose condensation temperature for non-interacting bosons (dots) and strongly interacting bosons (solid line) as a function of number density $(n)$ for bosons in a sc lattice. Here $W$ is the half-band-width.

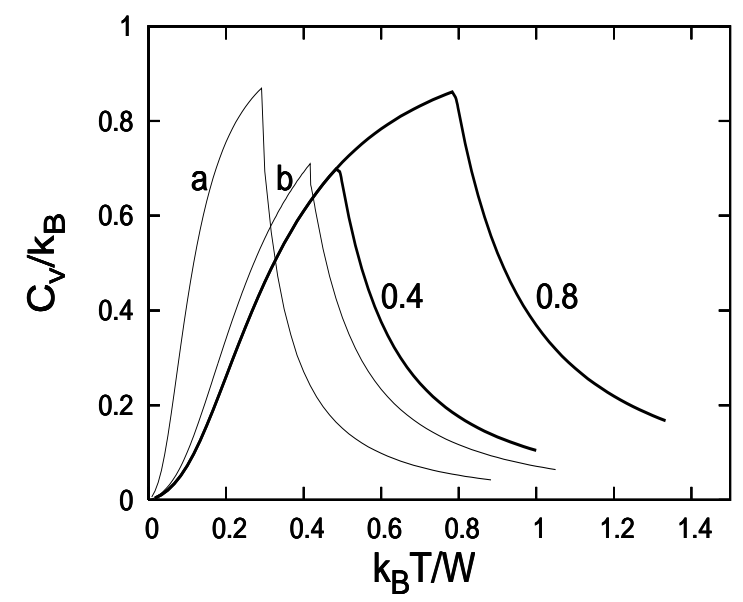

FIG. 2. Temperature variation of specific heat per site for non-interacting bosons (thick lines) in a sc lattice for two values of $n$ (0.4 and 0.8). Thin lines are for strongly interacting bosons, (a) $n=0.8$ and (b) $n=0.4$. 


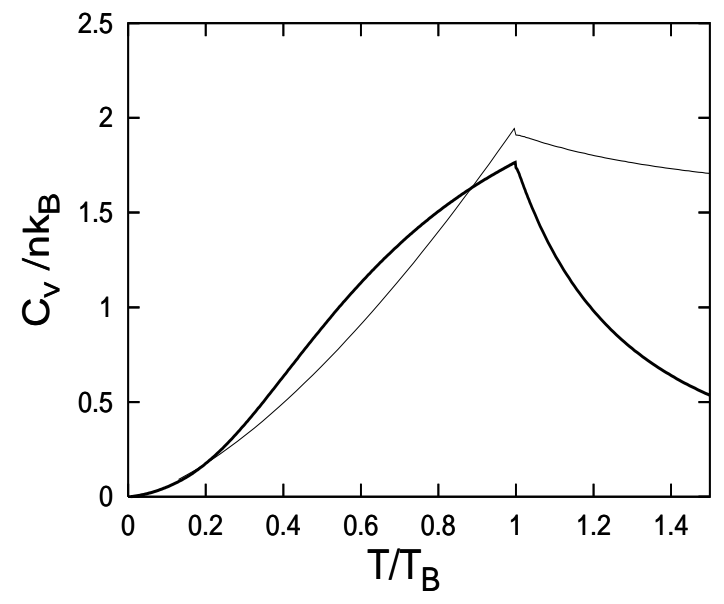

FIG. 3. Temperature variation of specific heat per particle for non-interacting bosons in a sc lattice (thick line) and for free bosons in a three dimensional box.

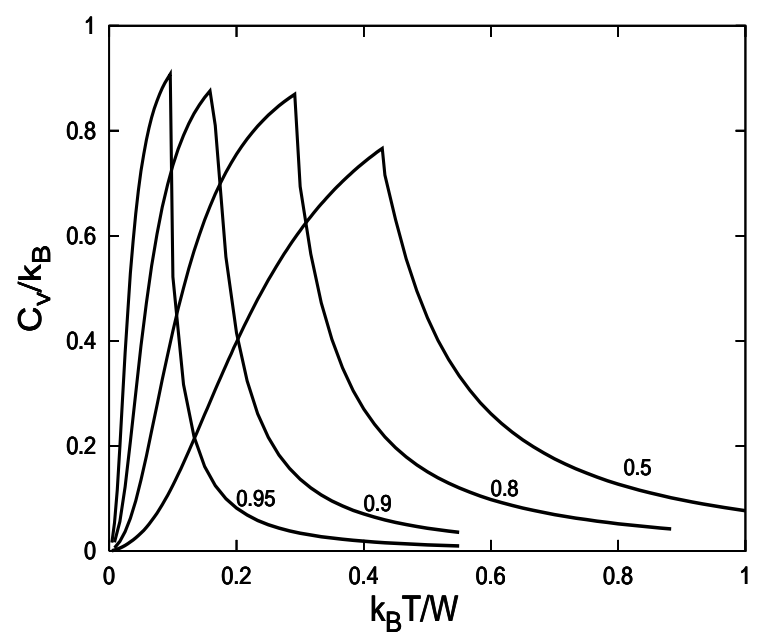

FIG. 4. Specific heat (per site) of Tonks gas in a sc lattice for several values of $n$ shown on the curves. 